Check for updates

Cite this: RSC Adv., 2017, 7, 50389

\title{
Carbon nanodots crosslinked photoluminescent alginate hydrogels $\uparrow$
}

\author{
Rangana Wijayapala, (D) Seyed Meysam Hashemnejad and Santanu Kundu (D)*
}

\begin{abstract}
Alginate hydrogels are commonly used in biomedical applications and these hydrogels are usually prepared by ionic or covalent crosslinking. This study reports a new synthesis route for preparing photoluminescent alginate hydrogels using amine functionalized carbon nanodots (CNDs). CNDs with amine functional groups have been synthesized from ortho, para, and meta phenylenediamine by using the solvothermal method. The nanodots display yellow, red, and blue color when excited with a UV light with the wavelength of $365 \mathrm{~nm}$. Amine groups on the CNDs form chemical crosslinks with alginate chains leading to the formation of a crosslinked network. The sol-to-gel transition has been monitored by using in situ shear rheometry. The CNDs crosslinked hydrogels maintain their fluorescent activity in a phosphatebuffered saline solution for a prolonged duration. Stable hydrogels with photoluminescent activity will have potential applications in metal ions sensing, drug delivery, and other biological applications.
\end{abstract}

Received 3rd September 2017

Accepted 23rd October 2017

DOI: $10.1039 / \mathrm{c} 7 \mathrm{ra0} 9805 \mathrm{~g}$

rsc.li/rsc-advances

delivery by utilizing CNDs coated alginate hydrogels. ${ }^{15,16}$ If CNDs are physically absorbed on ionically crosslinked alginate beads, the CNDs can leach out in a biological medium and diminish the benefit of using CNDs.

Hydrogels, particularly, that are synthesized from natural polymers such as collagen, polysaccharides have been investigated for many applications because of their bio compatibility, low toxicity, and easy synthesis routes. ${ }^{17-21}$ Alginates are polysaccharides extracted from brown algae. They are linear copolymers composed of $\beta$-D-mannuronic acid (M) and $\alpha$-L-guluronic acid (G) having different stereochemistry. ${ }^{22}$ Several experimental and computational studies revealed that only the G-G residues form ionic bonding with the divalent cations, for example, $\mathrm{Ca}^{2+}$, to form a crosslinked network. ${ }^{23-25}$ However, the mechanical properties of ionic crosslinked alginate hydrogels can undergo changes inside a physiological media containing salts. ${ }^{26}$ Here, charged species can alter the ionic crosslinking of the alginate gels leading to mechanical failure of the gels. ${ }^{26}$ Such degradation can be an issue for the application of these gels in biomedical applications such as drug delivery or tissue engineering. This problem can be overcome by chemically crosslinking the alginate chains. ${ }^{27}$ Presence of carboxylic acid groups in alginate chains allows one to form chemical crosslinks via amidation reaction with diamine molecules.

In many applications, such as in drug delivery, cell microencapsulation for the delivery of biopharmaceutics, and tissue engineering, monitoring the stability of hydrogels inside the biological media is important. This can be potentially achieved by taking the benefits of the fluorescence activity of CNDs and the biocompatibility of alginate gels. Towards that goal, here we present a facile synthesis route for CNDs mediated crosslinking of alginate gels. Multicolor photoluminescent CNDs have

Dave C. Swalm Swalm School of Chemical Engineering, Mississippi State University, Mississippi State, MS 3975, USA. E-mail: santunukundu@che.msstate.edu

$\dagger$ Electronic supplementary information (ESI) available: TEM image of CNDs, FTIR spectra of CNDs and precursor materials, shear rheology to capture in situ gelation and gel fracture, and stability of gels in phosphate-buffered saline solution. See DOI: 10.1039/c7ra09805g 
synthesized by using solvothermal synthesis method from ortho-, para-, and meta-phenylenediamine..$^{28,29}$ These CNDs are inherently amine functionalized and are used for chemical crosslinking of alginate chains. Such nanoparticle-mediated synthesis of alginate hydrogels has not been reported widely in the literature. The synthesized hydrogels display the similar photoluminescence of the CNDs, it was incorporated with. Fluorescence activity of these gels remained stable for a prolonged duration in a phosphate-buffered saline media, in comparison to the dissociation of ionically crosslinked alginate gels. We anticipate that this novel fluorescence active CNDs mediated chemically crosslinked alginate hydrogels will openup novel biomedical and biosensing applications.

\section{Experimental}

\section{Materials}

$o$-Phenylenediamine (99.5\%), $m$-phenylenediamine (99.0\%), $p$-phenylenediamine (99.0\%), $N$-(3-dimethylaminopropyl)- $N$ ethylcarbodiimide (EDC), 2-( $N$-morpholino)ethanesulfonic acid (MES), 1-hydroxybenzotriazole hydrate (HOBt), calcium phosphate $\left(\mathrm{CaHPO}_{4}\right)$, and glucono delta-lactone (GDL), $\mathrm{NaOH}(1 \mathrm{M})$, phosphate buffered saline solution $(\mathrm{pH}=7.4)$ and ethanol, were purchased from Sigma-Aldrich (St. Louis, MO). Sodium alginate was obtained from FMC BioPolymer (Protananal LF 20/ 40, Batch \#G5703401). All chemicals were used as received without further purification. Deionized water (resistivity of $18.2 \mathrm{~m} \Omega$ ) was used for this study.

\section{Synthesis and characterization of CNDs}

$0.5 \mathrm{~g}$ of phenylenediamine $(o, m$, or $p$ ) was dissolved in $50 \mathrm{~mL}$ of ethanol. The solution was heated inside a PTFE lined autoclave at $180{ }^{\circ} \mathrm{C}$ for $12 \mathrm{~h}$. After the reaction media was cooled down to the room temperature, a sequence of centrifugation steps was used to separate the CNDs as a precipitate. CNDs (the precipitate) were then dried using a freeze dryer to remove the ethanol. CNDs obtained from $o, m$, or $p$-phenylenediamine have been labeled as $o$-CNDs, $m$-CNDs, and $p$-CNDs, respectively. These CNDs were examined for the fluorescence activity and were used for alginate gel formation after dissolving in DI water in an appropriate quantity. Fluoromax-4 spectrometer (HORIBA) was used to evaluate the photoluminescence activity of the CNDs and CNDs crosslinked alginate hydrogels. The FTIR spectra were recorded by using a Thermo Nicolet Nexus 6700 instrument. Thermo Scientific K-Alpha XPS system equipped with a monochromatic X-ray source at $1486.6 \mathrm{eV}$ corresponding to the $\mathrm{Al} \mathrm{K} \alpha$ line was used for the XPS experiments. XPS experiments were conducted on the freeze-dried CNDs. To investigate the morphologies of the CNDs, transmission electron microscopy (TEM) and atomic force microscopy (AFM) characterizations were conducted using JEOL $2100(200 \mathrm{kV})$ TEM and Bruker dimension icon AFM.

\section{Preparation of alginate hydrogels}

Photoluminescent alginate hydrogels were prepared by two types of crosslinking, CNDs crosslinked (chemical) and ionically crosslinked using divalent $\mathrm{Ca}^{2+}$. For crosslinking with CNDs, alginate powder was dissolved in a buffer solution ( $\mathrm{pH} \sim 6.0$ ) using an overhead mixer and the alginate concentration was maintained at $10 \mathrm{mg} \mathrm{mL}{ }^{-1}$. EDC and HOBt were used in order to activate the carboxylic groups on alginate chains. Moles of $\mathrm{COOH}$ group was determined by considering the molecular weight of alginate as $260000 \mathrm{~g} \mathrm{~mol} \mathrm{~m}^{-1} .^{30}$ EDC : HOBt : COOH molar ratios were selected as 1.0 : 1.0 : 1.0 . After obtaining a homogeneous alginate solution, EDC was added and was then mixed for $60 \mathrm{~min}$. Afterwards, HOBt was added and was mixed for additional $30 \mathrm{~min}$. In the last step, amine functionalized CNDs was added maintaining the concentration of $1 \mathrm{mg} \mathrm{mL}^{-1}$. The solution was further mixed for 1 min prior to either immediately transferring to the rheometer for in situ gelation or casting in a Petri-dish.

The synthesized gels were also analyzed using scanning electron microscopy (SEM) using a JEOL 6500F Field Emission SEM. Here, gel samples were quickly frozen by dipping in liquid nitrogen. The frozen hydrogels were then fractured with a sharp scalpel. Fractured gels were transferred to a freeze dryer and freeze-dried until all the solvent (water) sublimed. ${ }^{31}$ A very lightweight dry gel was obtained after $48 \mathrm{~h}$ of freeze-drying. This sample was then investigated in SEM.

For ionically crosslinked alginate gels with CNDs, an alginate stock solution (10 $\mathrm{mg} \mathrm{mL} \mathrm{mL}^{-1}$ ) in DI water was first prepared. $\mathrm{CaHPO}_{4}$ powder was added to the alginate solution and was mixed for about $5 \mathrm{~min}$. GDL and CNDs $\left(1 \mathrm{mg} \mathrm{mL}{ }^{-1}\right)$ were then added and the solution was mixed for $1 \mathrm{~min}$. The solution was then casted in Petri-dish for swelling measurements. For this study, $\left[\mathrm{Ca}^{2+}\right]$ was chosen as $12.5 \mathrm{mM}$. GDL/Ca ${ }^{2+}$ molar ratio was selected as $3 .^{30}$

\section{Rheological characterization}

Shear rheological measurements were conducted using a stress-controlled rheometer, DHR2 (TA Instruments). A $20 \mathrm{~mm}$ parallel plate with solvent trap geometry was used. The gap between plates was selected as $1.5 \mathrm{~mm}$. A rough sand paper (grit \#60) was attached to both plates to minimize the slippage at the interface between plates and the gel during rheological experiments. In situ gelation was monitored by conducting a time sweep experiment with strain amplitude $\gamma_{0}=0.1 \%$ at an angular frequency $\omega=1 \mathrm{rad} \mathrm{s}^{-1}$. Frequency sweep (at $\gamma_{0}=0.1 \%$ and $\omega=0.1-20 \mathrm{rad} \mathrm{s}^{-1}$ ) followed by strain sweep (at $\omega=0.5 \mathrm{rad} \mathrm{s}^{-1} \gamma_{0}=0.1-300 \%$ ) experiments were performed on the gel samples. All measurements were performed at room temperature.

\section{Evaluation of hydrogels stability and swelling}

The prepared ionic and chemically crosslinked photoluminescent hydrogels were placed in a phosphate-buffered saline solution $(\mathrm{pH}=7.4)$ to determine the change in swelling behavior as a function of time. Gels were cut in small cubes prior to immersing in saline solution in $20 \mathrm{~mL}$ vials. Gels were removed from the solution after known time interval to measure their mass $(\mathrm{m})$. The normalized weight was determined as $m / m_{0}$, where $m_{0}$ is the initial mass of the gel cube. 


\section{Results and discussion}

\section{Photoluminescent carbon nanodots}

Phenelenediamine was particularly selected as the starting material to obtain amine functionalized CNDs. These nanodots have a diameter of $\sim 10 \mathrm{~nm}$, as observed in TEM and AFM images (Fig. S1 $\dagger$ ). The $p$-CNDs are slightly bigger than $o$-CNDs, which in turn are bigger than $m$-CNDs. After synthesis using solvothermal methods ${ }^{28}$ fluorescence properties of $o, m$, and $p$-CNDs were investigated in aqueous media. Fig. 1 displays the emission spectra for a single excitation wavelength of $\lambda=$ $365 \mathrm{~nm}$. $m$-CNDs exhibited the emission maxima at $\lambda=$ $455 \mathrm{~nm}$, which is in the range of blue color in visible spectrum. Ortho and para emission maxima were found at $\lambda=555 \mathrm{~nm}$, $675 \mathrm{~nm}$, corresponding to yellow and orange-red color, respectively. FTIR analysis was conducted to investigate the nature of functional groups on these CNDs. As shown in Fig. S2a, $\uparrow$ the FTIR spectra for $o-, m$, and $p$-CNDs exhibited similar chemical composition. The peaks at $\approx 3356 \mathrm{~cm}^{-1}$ and at $\approx 1620 \mathrm{~cm}^{-1}$ are primarily due to amine functional group (N-H stretching and $\mathrm{N}-\mathrm{H}$ bending). Several distinct peaks at about $\approx 2924 \mathrm{~cm}^{-1}$ and $\approx 1497 \mathrm{~cm}^{-1}$ appeared in the CNDs spectra compared to that of the pure phenylenediamine (Fig. S2b $\dagger$ ). These peaks are likely due to the decomposition, intermolecular cyclization, and condensation reactions occurred during the formation of CNDs. ${ }^{28}$ The XPS spectra and the relative $\mathrm{C}, \mathrm{N}$, and $\mathrm{O}$ content for the CNDs are shown in Fig. S3. $\dagger$ Similar to that reported in the literature, the $\mathrm{N}$

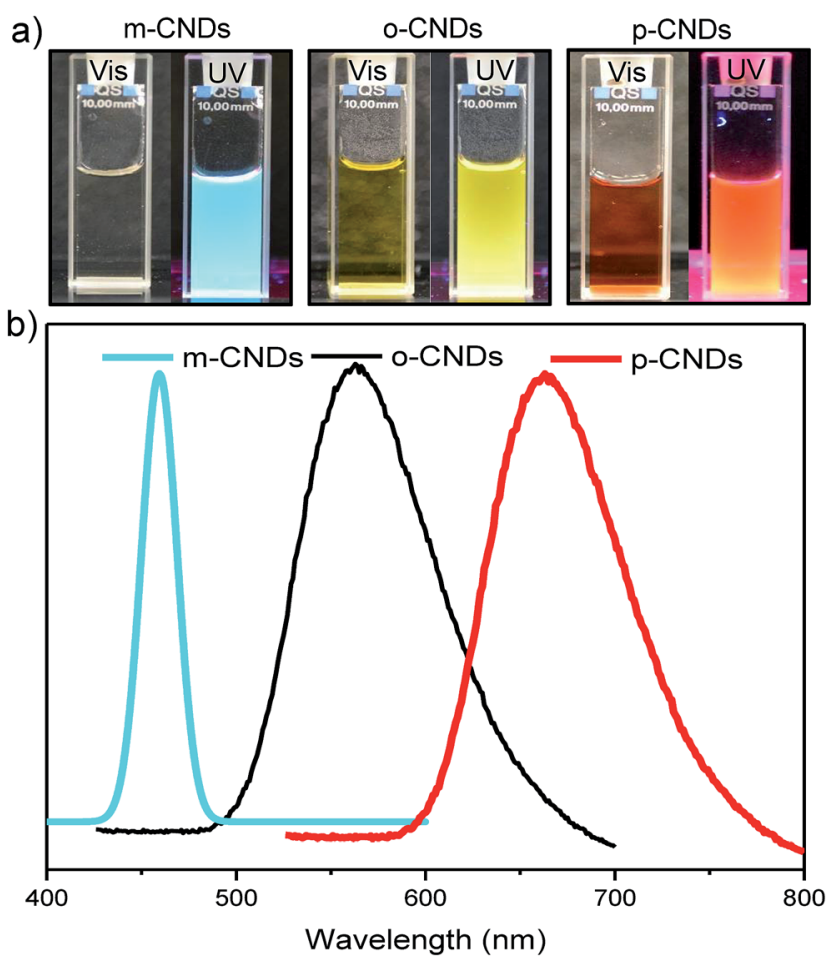

Fig. 1 (a) Comparison of CNDs color under natural light and UV light (left to right: $m$-CNDs, $o-C N D s$ and $p$-CNDs). (b) Fluorescence emission spectra of $m$-CNDs, $O-C N D s$ and $p$-CNDs $\left(\lambda_{\mathrm{Ex}}=365 \mathrm{~nm}\right)$. content is highest in $p$-CNDs. ${ }^{28} m, o$, and $p$-CNDs showing three different emission maxima (blue, yellow and red) have also been reported in the literature. ${ }^{28}$ These three different colors are likely due to the different sizes (Fig. S1†) and nitrogen percentages (Fig. S3†) in the CNDs.

\section{Alginate gel formation}

Alginate polymer chains consist of the carboxylic functional groups $(\mathrm{COOH})$. These carboxylic functional groups are used to crosslink (either ionically or chemically) to obtain alginate gels. ${ }^{27}$ Ionically crosslinked alginate gels are most common, and the gelation is mostly conducted using divalent calcium salts, which form ionic bonding with the carboxylic functional groups. One $\mathrm{Ca}^{2+}$ ion can potentially bond with two chains (mostly GG blocks) leading to crosslinking. In fact, multiple $\mathrm{Ca}^{2+}$ ions form ionic bond with alginate chains to form junction zones. $^{30}$

CNDs synthesized here contain amine functional groups on the surface. As shown in Fig. 2, the carboxylic groups of alginate chains have been activated by using EDC and HOBt. ${ }^{27}$ These chains then react with the primary amine of the CNDs forming covalent bonds (amidation reaction). Since the CNDs can have multiple amine groups, those can theoretically associate with multiple chains. However, the size of CNDs $(\sim 10 \mathrm{~nm})$ and the persistence length of alginate chains $(\sim 15 \mathrm{~nm})^{30}$ will dictate the number of chains that can link with a single CND. Because of large persistence length, it is unlikely that the alginate chains wrap around the CNDs. Although, we have not conducted any scattering study to investigate the structure of these gels, we can anticipate that egg-box junction zones are not present here. Rather, the crosslinking is random throughout the sample, similar to that observed for chemically crosslinked alginate gels. ${ }^{27}$ SEM (Fig. S4 $\dagger$ ) captures the gel morphology similar to that observed in literature for alginate gels. ${ }^{31}$ There are reports on chemically crosslinked alginate gels by using diamines, ${ }^{27}$ but nanoparticle-mediated gelation of alginate has not been reported. Our results also differ from the earlier reports on CND containing hydrogels, ${ }^{14}$ as CNDs in our system participate in the gelation process as the crosslinkers. Modulus of CNDs crosslinked alginate gels can be readily varied by changing the concentration and types of CNDs.

\section{Rheological characterization of CNDs-alginate hydrogels}

The sol-to-gel transition of CNDs crosslinked hydrogels was monitored in situ on a rheometer. Dynamic rheological measurement was conducted to probe the evolution of shear moduli during the transition from an aqueous solution to a gel state (Fig. S5 $\dagger$ ). $G^{\prime}$ (storage modulus) and $G^{\prime \prime}$ (loss modulus) increased with time and reached a plateau after $\sim 4 \mathrm{~h}$ for $o^{-}$ CNDs-alginate gel, however, increase in $G^{\prime}$ was not significant after about $2 \mathrm{~h}$. After $4 \mathrm{~h}$ of gelation $G^{\prime}$ is more than two orders of magnitude higher than $G^{\prime \prime}$, indicating the completion of gelation process.

After in situ gelation on the rheometer, frequency and strain sweep experiments were conducted. Fig. 3a displays $G^{\prime}$ and $G^{\prime \prime}$ as a function of frequency for $o^{-}, m^{-}$, and $p$-CNDs-alginate gels. 


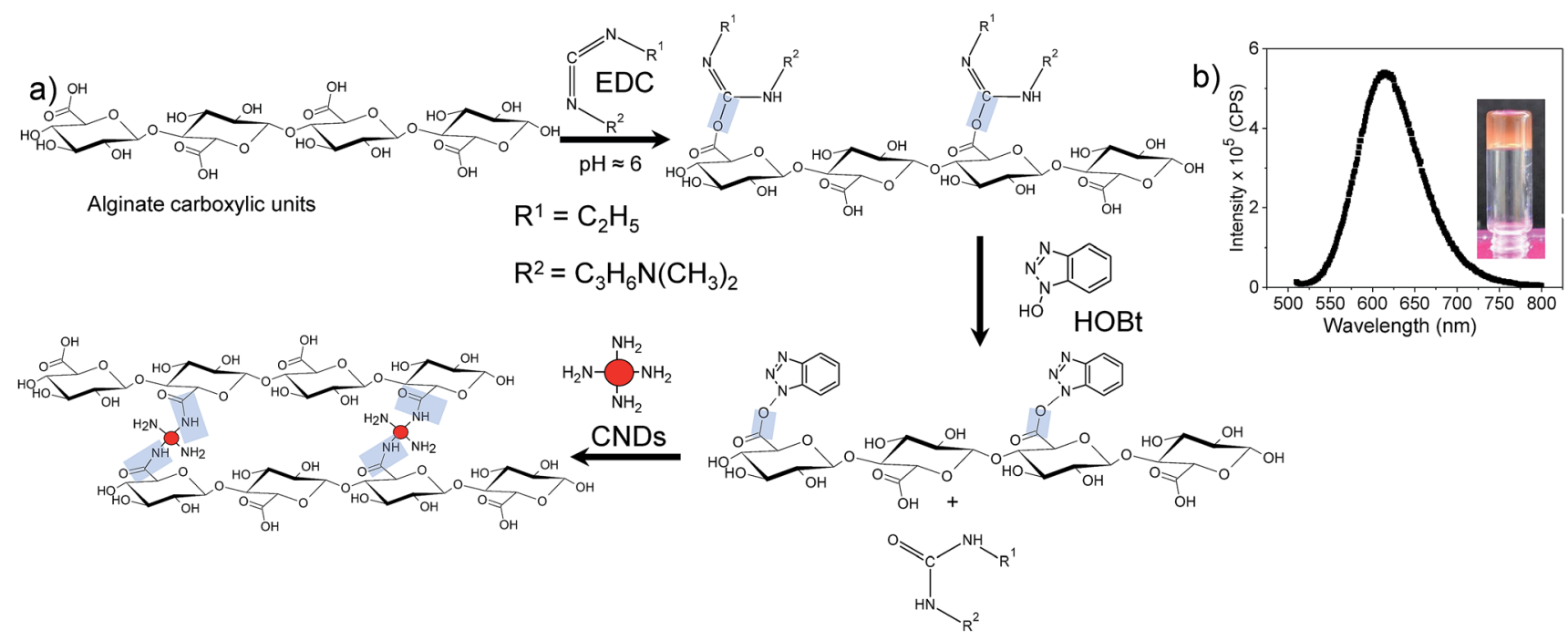

Fig. 2 (a) Reaction scheme for the CNDs crosslinked alginate gels and (b) fluorescence emission spectra of $p$-CNDs alginate gel ( $\lambda_{\mathrm{Ex}}=500 \mathrm{~nm}$ ).
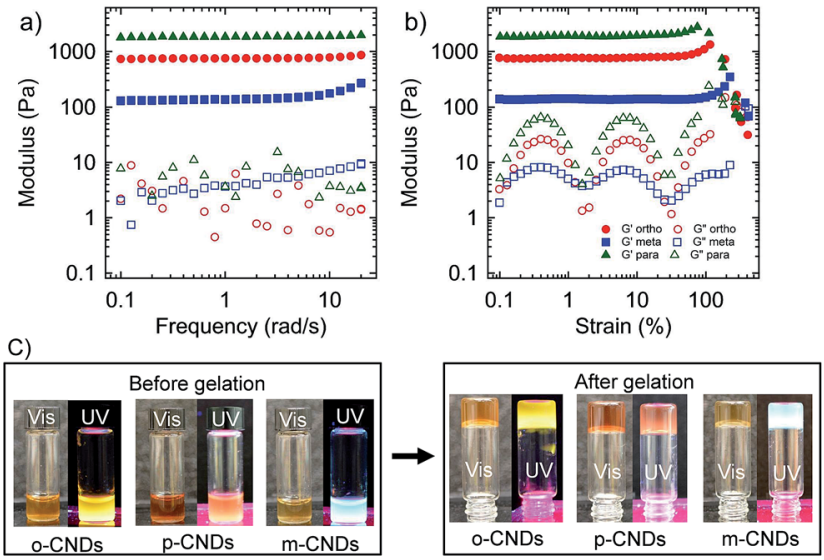

Fig. 3 Rheological responses of the CNDs crosslinked alginate hydrogels. (a) Frequency and (b) strain sweep results on the gels formed in situ on the rheometer plate. (c) Vial inversion tests of aqueous solutions and after forming gels. Color of the gels under natural and UV lights are also shown.

In all cases, elastic moduli were found to be almost independent of frequency over the range investigated here. Interestingly, the mechanical properties of hydrogels depend on the type of CNDs used. For example, the $p$-CNDs-alginate gel has the highest shear modulus, whereas, the $m$-CNDs-alginate gel has the lowest one. The difference in $G^{\prime}$ for a similar alginate and CNDs concentrations has been found to be as high as ten times. Shear modulus is directly linked to the crosslink density and higher modulus represent higher crosslink density. At this stage, the exact reason behind the differences in shear modulus for different CNDs is not known, but can be hypothesized to be linked to the difference in chemical functionalities of CNDs, particularly, the number and accessibilities of the amine groups. XPS analysis on CNDs revealed that the $p$-CNDs have the highest nitrogen content (Fig. S3†), whereas $m$-CNDs contains the lowest amount of nitrogen.$^{28}$ In addition, the size of the nanodots can play a role, as the CNDs have dimensions closer to the persistence length of the chains. As discussed above (also see Fig. S1†), $p$-CNDs have the largest particle size, whereas, $m$-CNDs have the smallest one, similar to that reported earlier. ${ }^{28}$

Subsequent to the frequency sweep test, large amplitude oscillatory shear experiments were performed. Fig. 3b displays $G^{\prime}$ and $G^{\prime \prime}$ of these gels as a function of shear strain amplitude. In general, CNDs-alginate hydrogels display strain stiffening behavior, similar to that observed for ionically crosslinked gels. ${ }^{30}$ The strain stiffening behavior was more pronounced for the gels with the lowest modulus. For example, $G^{\prime}$ in $m$-CNDsalginate gel increased more than two times before the material failed in shear-mode. This strain-stiffening behavior can be attributed to the stiffness of alginate chains (semi-flexible), as the junctions zones typical to ionically crosslinked chains are not expected to be present here. The drop of $G^{\prime}$ at large strain was due to the cohesive fracture of the network in all CNDsalginate gel (Fig. S6 $\dagger^{\dagger}$ ).

In addition to CNDs crosslinked hydrogels, ionically crosslinked gels with CNDs were also prepared by adding the CNDs to the pre-gel solution. For these samples, once the gels form, the CNDs are present in the interstitial space of hydrogels and are not chemically linked with the alginate chains. In an earlier study, we have captured the gelation of ionically crosslinked alginate gels. ${ }^{30}$ For those samples, gelation time was about 6 to $8 \mathrm{~h}$, longer than that observed for CNDs crosslinked alginate gels, for similar alginate concentration.

\section{Gel stability}

For the application of these hydrogels in biomedical applications, the stability of these gels inside biological media is important. In this study, the phosphate-buffered saline solution was used to mimic the physiological conditions. Fluorescence activity of the hydrogels was examined after submerging the samples in the buffer solution for a known period of time. Both 
ionic and chemically crosslinked hydrogels with CNDs were used for this experiment. The leaching of CNDs in the solution was monitored by measuring the fluorescence intensity of the submerging solution as a function of time, as shown in Fig. 4. The normalized intensities are shown in Fig. 4, whereas, the actual intensities are shown in Fig. S7. $\uparrow$ For all samples, the fluorescence of the submerging solution increased initially indicating leaching of CNDs. However, for CNDs-alginate gels, after this initial increase in fluorescence, no further significant change was observed in first $8 \mathrm{~h}$. Even after $24 \mathrm{~h}$, CNDs crosslinked hydrogels maintained the fluorescence activity (Fig. S8†). However, for ionic alginates, the fluorescence intensity of the submerging media continued to increase with time, indicating continuous leaching of CNDs from the samples. After about $3 \mathrm{~h}$ most of the CNDs leached out in the media and the gels were no longer fluorescent.

Above results further suggest that in these ionically crosslinked alginate gels the CNDs were not chemically linked. Because of complete leaching of CNDs, these ionic hydrogels cannot be fluorescently tracked inside a biological medium after a short period of time. The CNDs crosslinked hydrogels were superior in that respect, as the CNDs leaching was not significant. This initial increase in fluorescence of the submerging media was caused by the leaching of a small amount unreacted CNDs trapped inside the hydrogels.

Swelling and degradation behavior of the hydrogels were investigated by submerging these gels in phosphate-buffered saline solution over a period of time. The increase of mass, represented by normalized mass $\left(m_{t} / m_{0}\right)$ was monitored as a function of time. As shown in Fig. 5, a small swelling of ionically crosslinked hydrogels was observed, i.e., $m_{t} / m_{0} \approx 2.2$,
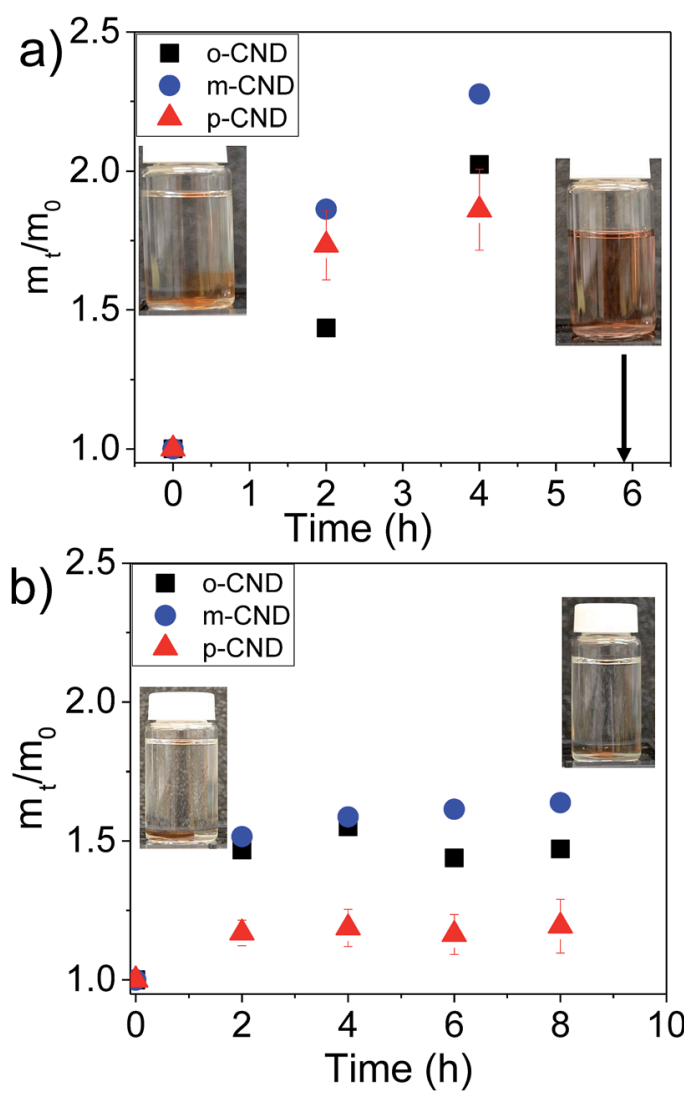

Fig. 5 Swelling behavior in phosphate-buffered saline solution. (a) Alginate with ionic crosslinking, (b) alginate crosslinked with CNDs.
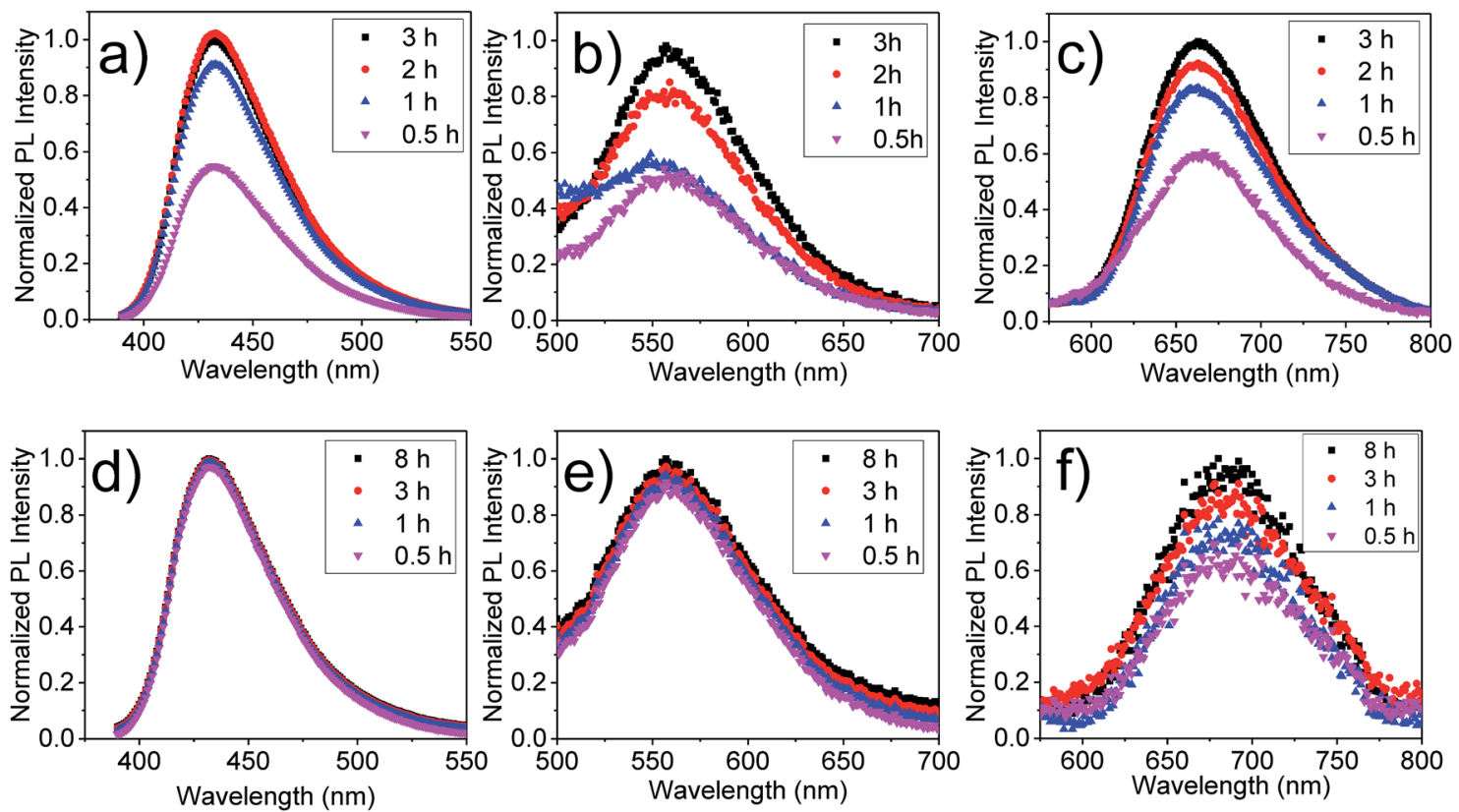

Fig. 4 Leaching of CNDs from hydrogels in the phosphate-buffered saline solution as a function of time monitored by using fluorescence spectroscopy of the submerging media. Normalized intensities are shown here. (a), (b) and (c) lonic crosslinked alginate hydrogels with $m$-CNDs, $o$-CNDs and $p$-CNDs, respectively. (d), (e) and (f) Chemically crosslinked alginate hydrogels with $m$-CNDs, o-CNDs and $p$-CNDs, respectively. Scatter in (f) is due to low fluorescence of the media. 
after waiting for 4 hours. However, as we waited longer, the gels started to disintegrate. After $5 \mathrm{~h}$, hydrogels were totally dissociated. Correspondingly, the color of the saline solution changed according to the type of CNDs present within the hydrogel (Fig. S9 $\dagger$ ). In contrast, the swelling of CNDs crosslinked alginates was much smaller $\left(m_{t} / m_{0} \approx 1.5\right)$. These hydrogels have been found to be very stable and have not shown any deterioration even after $8 \mathrm{~h}$.

As shown for most of the CNDs in the literature, the CNDs and the alginate gels consisting of CNDs presented here display fluorescence with the excitation of UV light. However, for bioimaging purpose it will be beneficial to use higher wavelength or lower energy excitation, as higher energy excitation wave length can possibly cause some harmful effects to the living cells. Therefore, $p$-CNDs and $p$-CNDs-alginate gels fluorescence activity was investigated over the wavelength range of $500 \mathrm{~nm}$ to $600 \mathrm{~nm}$, as shown in Fig. 6. $m$ and $o$-CNDs were not considered here, as these CNDs cannot be exited above the wavelength of $500 \mathrm{~nm}$. $p$-CNDs in water have shown its maximum fluorescence intensity when excited at $520 \mathrm{~nm}$. In contrast, the gels exhibited the highest intensity at $500 \mathrm{~nm}$ excitation. The gel has also shown a considerable amount of fluorescence activity upto $560 \mathrm{~nm}$ excitation. Therefore, this can be the feasible excitation wavelength region for bioimaging applications. ${ }^{32}$
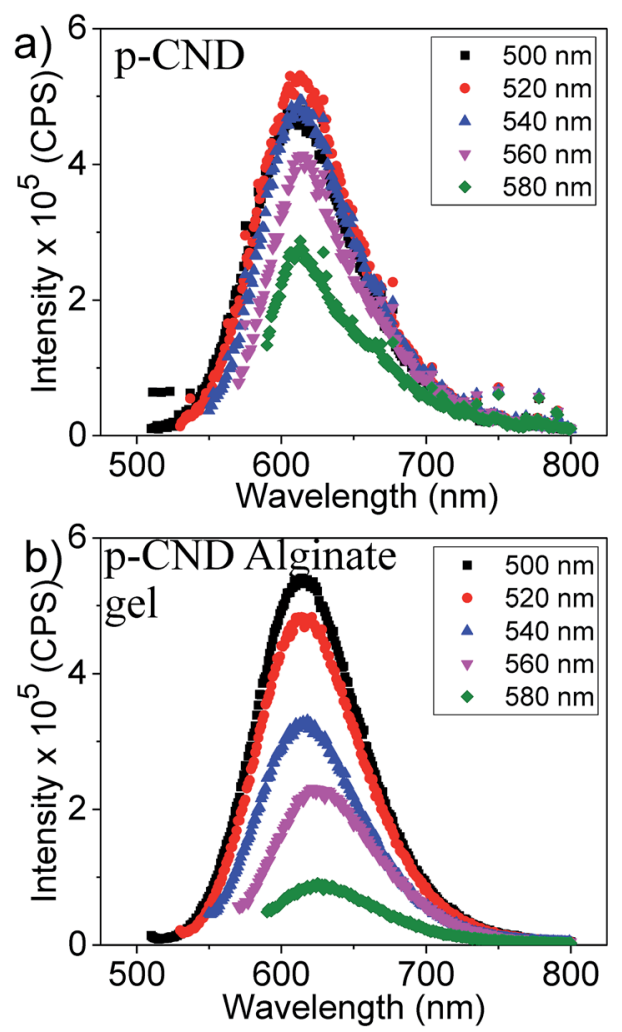

Fig. 6 (a) Fluorescence emission spectra of the $p$-CND and (b) $p$-CND crosslinked alginate gels excited in the wavelength range of 500$580 \mathrm{~nm}$.

\section{Conclusion}

The study has shown a facile synthesis strategy for chemically crosslinked fluorescence active alginate hydrogels with CNDs by using amidation reaction. The shear-modulus has been found to be dependent on the type of CNDs. Similar to the CNDs, these hydrogels are fluorescent active. The gels crosslinked with $o$-CNDs and $m$-CNDs display fluorescence activity when excited using UV light. In contrast, the gels with $p$-CNDs can be excited over a broader wavelength region. The CNDs crosslinked hydrogels are stable in a phosphate-buffered saline stable, in comparison to the conventional ionically crosslinked hydrogels, which dissociate over time. The CNDs crosslinked gels remain fluorescent active for a prolonged duration. These stable and photoluminescent hydrogels have potential applications in drug delivery and tissue scaffolding.

\section{Conflicts of interest}

There are no conflicts to declare.

\section{Acknowledgements}

This research was supported in part by the National Science Foundation's Experimental Program to Stimulate Competitive Research (EPSCoR) under Cooperative Agreement No. IIA1430364. SK and SMH also would like to acknowledge support from the National Science Foundation CAREER Award (DMR-1352572).

\section{References}

1 J. Kido and Y. Okamoto, Chem. Rev., 2002, 102, 2357-2368.

2 J. C. G. Bunzli and C. Piguet, Chem. Soc. Rev., 2005, 34, 10481077.

3 F. Wang and X. Liu, Acc. Chem. Res., 2014, 47, 1378-1385.

4 Y. Rong, C. Wu, J. Yu, X. Zhang, F. Ye, M. Zeigler, M. E. Gallina, I. C. Wu, Y. Zhang, Y.-H. Chan, W. Sun, K. Uvdal and D. T. Chiu, ACS Nano, 2013, 7, 376-384.

5 A. Patra, C. G. Chandaluri and T. P. Radhakrishnan, Nanoscale, 2012, 4, 343-359.

6 D. Yao, S. Zhao, J. Guo, Z. Zhang, H. Zhang, Y. Liu and Y. Wang, J. Mater. Chem., 2011, 21, 3568-3570.

7 A. Jaiswal, S. S. Ghosh and A. Chattopadhyay, Chem. Commun., 2012, 48, 407-409.

8 C. Ding, A. Zhu and Y. Tian, Acc. Chem. Res., 2014, 47, 20-30. 9 M. Zheng, Z. Xie, D. Qu, D. Li, P. Du, X. Jing and Z. Sun, ACS Appl. Mater. Interfaces, 2013, 5, 13242-13247.

10 V. Strauss, J. T. Margraf, K. Dirian, Z. Syrgiannis, M. Prato, C. Wessendorf, A. Hirsch, T. Clark and D. M. Guldi, Angew. Chem., Int. Ed., 2015, 54, 8292-8297.

11 X. Cui, L. Zhu, J. Wu, Y. Hou, P. Wang, Z. Wang and M. Yang, Biosens. Bioelectron., 2015, 63, 506-512.

12 H. Dai, Y. Shi, Y. Wang, Y. Sun, J. Hu, P. Ni and Z. Li, Sens. Actuators, B, 2014, 202, 201-208.

13 S. Liu, N. Zhao, Z. Cheng and H. Liu, Nanoscale, 2015, 7, 6836-6842. 
14 P. Zhang, W. Li, X. Zhai, C. Liu, L. Dai and W. Liu, Chem. Commun., 2012, 48, 10431-10433.

15 S. Majumdar, G. Krishnatreya, N. Gogoi, D. Thakur and D. Chowdhury, ACS Appl. Mater. Interfaces, 2016, 8, 3417934184.

16 N. Gogoi and D. Chowdhury, J. Mater. Chem. A, 2014, 2, 4089-4099.

17 E. M. Ahmed, J. Adv. Res., 2015, 6, 105-121.

18 J. Baier Leach, K. A. Bivens, C. W. Patrick Jr and C. E. Schmidt, Biotechnol. Bioeng., 2003, 82, 578-589.

19 J. A. Rowley, G. Madlambayan and D. J. Mooney, Biomaterials, 1999, 20, 45-53.

20 A. D. Augst, H. J. Kong and D. J. Mooney, Macromol. Biosci., 2006, 6, 623-633.

21 C. K. Kuo and P. X. Ma, Biomaterials, 2001, 22, 511-521.

22 O. Smidsrød and G. Skjåk-Braek, Trends Biotechnol., 1990, 8, 71-78.

23 L. Li, Y. Fang, R. Vreeker, I. Appelqvist and E. Mendes, Biomacromolecules, 2007, 8, 464-468.
24 W. Plazinski and M. Drach, J. Phys. Chem. B, 2013, 117, 12105-12112.

25 G. T. Grant, E. R. Morris, D. A. Rees, P. J. C. Smith and D. Thom, FEBS Lett., 1973, 32, 195-198.

26 M. S. Shoichet, R. H. Li, M. L. White and S. R. Winn, Biotechnol. Bioeng., 1996, 50, 374-381.

27 P. Eiselt, K. Y. Lee and D. J. Mooney, Macromolecules, 1999, 32, 5561-5566.

28 K. Jiang, S. Sun, L. Zhang, Y. Lu, A. Wu, C. Cai and H. Lin, Angew. Chem., Int. Ed., 2015, 54, 5360-5363.

29 M. Vedamalai, A. P. Periasamy, C. W. Wang, Y. T. Tseng, L. C. Ho, C. C. Shih and H. T. Chang, Nanoscale, 2014, 6, 13119-13125.

30 S. M. Hashemnejad and S. Kundu, J. Polym. Sci., Part B: Polym. Phys., 2016, 54, 1767-1775.

31 Y. Zhao, S. Gao, S. Zhao, Y. Li, L. Cheng, J. Li and Y. Yin, Mater. Sci. Eng., C, 2012, 32, 2153-2162.

32 S. Lu, L. Sui, J. Liu, S. Zhu, A. Chen, M. Jin and B. Yang, Adv. Mater., 2017, 29, 1603443. 